\title{
Percepção de fala em idosos: análise dos erros
}

\author{
Speech perceptionintheedderly: errorsanalysis
}

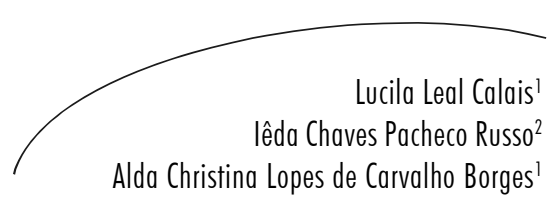

Resumo

Objetivo: analisar os erros apresentados em um teste de fala na presença de ruído competitivo de idosos. Método: foi realizado estudo retrospectivo com levantamento de dados de prontuário de 55 idosos divididos em grupo controle (GC) e grupo estudo (GE), considerando os testes Índice Percentual de Reconhecimento de Fala (IPRF) e Fala com Ruído (F/R). Resultado: as trocas articulatórias foram mais frequentes no GE e no teste $F / R$. De maneira geral, ocorreram mais omissões de fonemas na posição inicial $(/ \mathrm{p} / \mathrm{e} / \mathrm{b} /)$ e final (/s/) dos vocábulos, principalmente com o ruído. Houve omissões de vocábulos somente no GE. Os vocábulos mais trocados no GC foram "rir", "dil”, "lhe", "faz" e "rol”, e no GE, "rir”, "dil”, "lhe”, "faz", "pus" e "rol”. Conclusão: a presença de limiares tonais normais não impediu que ocorressem erros no reconhecimento de fala e o ruído interferiu negativamente neste reconhecimento, principalmente no idoso com perda auditiva. Os fonemas / $\mathrm{p} /, / \mathrm{b} / \mathrm{e} / \mathrm{s} /$ foram os mais frequentemente omitidos, principalmente na presença do ruído competitivo. Os vocábulos "rir", “dil”, "lhe”, "faz" e "rol” foram os mais trocados para os grupos avaliados, sobretudo na presença do ruído.

\section{Abstract}

Objective: To analyze the errors made in a speech test in the presence of background noise for the elderly. Method: We performed a retrospective study with data collected from medical records of 55 elderly patients divided into control group (CG) and study group (SG), considering the tests Percentage Index of Speech Recognition (PISR) and Speech in Noise (S/R). Result: articulatory exchanges were more frequent test in the $S G$ and in the /R test. Overall, there were more omissions of phonemes in initial position $(/ \mathrm{p} /$ and $/ \mathrm{b} /$ ) and final $(/ \mathrm{s} /)$ of words, especially with the noise. There were omissions of words only in the SG. The words more exchanged in the CG were "rir", "dil”, "lhe”, "faz" and "rol”, and in the SG, "rir", "dil”, "lhe", "faz", "pus" and "rol". Conclusion: the presence of normal pure tone thresholds did not

\footnotetext{
Universidade Federal de São Paulo. Curso de Pós-Graduação em Distúrbios da Comunicação Humana. São Paulo, SP, Brasil.

2 Pontifícia Universidade Católica de São Paulo. Departamento de Clínica Fonoaudiológica. São Paulo, SP, Brasil.

Correspondência / Correspondence

Lucila Leal Calais

E-mail: calais@ig.com.br
}

Palavras-chave: Idoso. Percepção Auditiva. Testes de Discriminação da Fala. Presbiacusia. 
prevent errors that occur in speech recognition and noise interfered negatively in this recognition, especially in the elderly with hearing loss. The phonemes / p / / b / and / s / were most frequently omitted, especially in the presence of noise. The words "rir", "dil", "lhe", "faz" and "rol" were the most confused in the groups, mainly in the presence of noise.

\section{INTRODUÇÃO}

A população de idosos está aumentando de maneira considerável ${ }^{1}$, e tem resultado numa maior frequência de estudos envolvendo o processo de envelhecimento e os problemas enfrentados pelos idosos. Desta forma, os resultados obtidos e as reflexões geradas por estes estudos permitirão formular propostas que solucionem os problemas de saúde desta população ou minimizem as dificuldades para uma melhora da qualidade de vida.

É de conhecimento geral que o envelhecimento acarreta a deterioração de várias funções do organismo e, dentre eles, a função auditiva. $\mathrm{O}$ termo "presbiacusia" surge então para definir a perda auditiva decorrente do envelhecimento do órgão auditivo.

A presbiacusia é descrita como uma perda auditiva bilateral para sons de alta frequência, geralmente acompanhada por uma perda desproporcional do reconhecimento da fala, sem história prévia de doença sistêmica ou auditiva severa, com início gradual e curso progressivo².

A perda auditiva é um dos problemas crônicos mais frequentemente encontrados nos idosos e tende a aumentar com a idade, acometendo $33 \%$ daqueles entre 65 e 74 anos, $45 \%$ das pessoas entre 75 e 84 anos e $62 \%$ das pessoas acima de 85 anos de idade ${ }^{3}$.

Para o idoso, a audição e seu funcionamento adequado têm papel fundamental para que ele possa se comunicar com os que estão a sua volta, se expressando e compreendendo o que lhe é dito e, assim, exercendo um papel ativo na sociedade.

A importância do funcionamento auditivo na vida do idoso é revelada na entrevista realizada na
Keywords: Aged, Auditory perception, Speech discrimination tests, Presbyacusis. rotina clínica, quando é relatada a queixa auditiva, mais especificamente quanto à compreensão $d a$ linguagem falada, sobretudo em situações nas quais há ruído no ambiente, fato já relatado em outros estudos ${ }^{2,4-6}$.

Diante da importância da função auditiva e das queixas apresentadas por esta população, faz-se necessária a avaliação deste órgão e, de preferência, com o uso de estímulos de fala com a presença de um estímulo competitivo, esta seria uma maneira mais real de avaliar a audição, refletindo e melhor documentando as possíveis dificuldades apresentadas pelos idosos no dia a dia ${ }^{7-10}$.

Vários estudos têm refletido sobre este tema ou demonstrado os efeitos prejudiciais do ruído na percepção da fala ${ }^{9,11-15}$. Entretanto, somente apresentam as pontuações dos erros no reconhecimento de fala, sendo os mesmos tratados com equivalência. A análise mais detalhada dos erros, acertos e omissões apresentados nos testes de reconhecimento de fala é pouco frequente na literatura, e, como observado em um estudo anterior ${ }^{16}$, perde-se um valioso material para compreendermos de maneira mais detalhada os aspectos envolvidos na percepção da fala, bem como a escolha de parâmetros na prescrição de características acústicas no processo de indicação e adaptação de próteses auditivas. Além disso, informações qualitativas sobre os erros cometidos poderiam servir para melhor orientar o sujeito idoso na reabilitação auditiva. Os estudos localizados foram os de Martini et al. ${ }^{17}$, que não utilizaram um ruído competitivo, e os de Schochat ${ }^{11}$ e Shrivastav et al. ${ }^{18}$, que fizeram uso de um estímulo competitivo juntamente com o material de fala. No estudo de Martini, foram revelados índices para trocas classificadas como completa distorção e de acordo com a categoria do fonema. Já o estudo de Schochat ${ }^{11}$ exemplificou 
as trocas ocorridas com maior significância e, na pesquisa de Shrivastav et al. ${ }^{18}$, há o relato quanto ao desempenho na tarefa de identificação do modo de articulação dos fonemas.

Diante do exposto, o presente trabalho tem como objetivo analisar qualitativa $e$ quantitativamente os erros apresentados em um teste de fala na presença de ruído competitivo de idosos.

\section{MÉTODO}

\section{Casuística}

Foi realizado estudo retrospectivo com o levantamento de dados de prontuário dos pacientes atendidos no Ambulatório da Disciplina de Distúrbios da Audição, do Departamento de Fonoaudiologia da UNIFESP, no período de setembro de 2004 a agosto de 2005. Este estudo obteve aprovação do Comitê de Ética em Pesquisa da UNIFESP, sob número 1784/07.

Os participantes foram esclarecidos sobre o objetivo deste estudo e convidados a participar do mesmo, o qual foi iniciado após a concordância e a assinatura do termo de consentimento livre e esclarecido. Foram respeitados todos os princípios éticos contidos na Resolução n ${ }^{\circ} 196 / 96{ }^{19}$ sobre ética em pesquisa com seres humanos e as orientações do Comitê de Ética em Pesquisa da UNIFESP.

Como critérios de inclusão, foram estabelecidos os seguintes itens: (a) idade mínima de 60 anos; (b) apresentar limiares audiométricos de $250 \mathrm{~Hz}$ a $8 \mathrm{kHz}$ dentro dos padrões de normalidade (até 25 dBNA); (c) apresentar audiometria de configuração descendente e simétrica conforme a seguinte descrição: limiar tonal abaixo de $30 \mathrm{~dB}$ no mínimo nas frequências de $6 \mathrm{kHz}$ e $8 \mathrm{kHz}$, caracterizando o início da perda auditiva pelo envelhecimento; limiar tonal médio das frequências de $500 \mathrm{~Hz}, 1 \mathrm{kHz}$, e $2 \mathrm{kHz}$ até $60 \mathrm{~dB}$, devido à intensidade máxima permitida pelo audiômetro para a realização dos testes de reconhecimento de fala sem e com ruído competitivo; (d) curva timpanométrica do tipo A (classificação segundo Jerger, 1970) ${ }^{20}$.

A casuística foi constituída de 55 indivíduos, sendo 49 (89,1\%) do grupo estudo (GE) e 6 (10,9\%) do grupo controle (GC). Todos os indivíduos eram idosos, com idade a partir de 60 anos, conforme estabelecido pela Lei ${ }^{\circ} 10.741 / 03^{21}$.

O GE foi composto de 43 mulheres (87,76\%) e seis homens $(12,24 \%)$ com idade mínima de 61 anos e máxima de 87 (média de 72,41 anos). Os indivíduos deste grupo apresentaram curva timpanométrica do tipo $\mathrm{A}^{21}$, perda auditiva neurossensorial simétrica descendente com limiar tonal abaixo de $30 \mathrm{~dB}$, no mínimo, nas frequências de $6 \mathrm{kHz}$ e $8 \mathrm{kHz}$ e limite máximo de $60 \mathrm{~dB}$ de limiar tonal médio das frequências de $500 \mathrm{~Hz}, 1 \mathrm{kHz}$ e $2 \mathrm{kHz}$.

O GC foi composto de seis mulheres com idade mínima de 60 anos e máxima de 72 (média de 65 anos). As idosas deste grupo também apresentaram curva timpanométrica do tipo A, mas os limiares audiométricos de $250 \mathrm{~Hz}$ a $8 \mathrm{kHz}$ estavam dentro dos padrões de normalidade (até 25 dB NA).

Foram excluídos do estudo os pacientes com quadros de alteração neurológica, com realização prévia de cirurgia otológica, exposição a ruído ocupacional, ingestão de medicação ototóxica e histórico de traumatismo craniano.

\section{Procedimento}

Neste trabalho foram analisados os seguintes dados dos prontuários dos pacientes:

- Dados de identificação: idade e sexo;

- Número de trocas e omissões apresentadas no Índice Percentual de Reconhecimento de Fala (IPRF) (anexo 1), bem como a descrição das mesmas para cada uma das orelhas;

- Número de trocas e omissões apresentadas no teste Fala com Ruído (F/R) (anexo 1), bem como a descrição das mesmas para cada uma das orelhas. 
Os testes dos casos analisados foram realizados em cabina acústica, sendo utilizados os audiômetros de dois canais GSI-64 e ORBITER-MADSEN 922, com fones supra-aurais TDH-39 adaptados ao coxim MX-41, devidamente calibrados, de acordo com a norma ISO 8253-1, $1989^{22}$.

O Índice Percentual de Reconhecimento de Fala (IPRF) e o teste Fala com Ruído (F/R) foram realizados com o auxílio do $\mathrm{CD}$ player da marca Toshiba modelo CDP 4147, acoplado ao audiômetro. Utilizou-se a segunda faixa do CD (volume 1) que acompanha o livro de Processamento Auditivo Central - Manual de Avaliação ${ }^{7}$, sendo acrescido ruído competitivo ipsilateral, do tipo Speech Noise no teste F/R. A relação sinal/ruído utilizada foi de $+5 \mathrm{~dB}$. Primeiramente, aplicou-se o teste na orelha direita e depois na orelha esquerda, sendo registrado o número total de vocábulos repetidos corretamente em cada um dos testes e, no caso de erro, a pesquisadora descreveu o que o sujeito informou ter compreendido.

Quanto à análise estatística, neste trabalho foi utilizado o teste não paramétrico Teste de Igualdade de Duas Proporções. O nível de significância adotado foi de 0,05 (5\%).

Foi realizada uma análise qualitativa dos acertos, erros e omissões nos testes IPRF e F/R, utilizando a Matriz de Confusão (MC), adaptada para o português brasileiro por Helou, Novaes ${ }^{16}$. $\mathrm{Na}$ Matriz de Confusão, se estabelecem os seguintes traços como base para a classificação das consoantes:

1. Vozeamento - em termos de articulação: as pregas vocais não vibram nos $/ \mathrm{p} /, / \mathrm{t} /, / \mathrm{k} /, / \mathrm{f} /$, /tS/, /s/, /S/ e vibram nos /b/, /d/, /g/, /v/, $/ \mathrm{dZ} /, / \mathrm{z} /, / \mathrm{Z} /, / \mathrm{m} /, / \mathrm{n} /$.

2. Nasalização para articular / $m / e / n /-\mathrm{A}$ ressonância nasal provoca uma diferença acústica e eles parecem levemente mais longos na duração que as fricativas ou plosivas, bem como algo mais intenso.

3. Friç̧ão - tipo de turbulência de ruído de fricção que distingue /f/, /tS/, /s/, /S/, /v/, /dZ/, /z/, /Z/ das consoantes /p/, /t/, /k/, $/ \mathrm{b} /, / \mathrm{d} /, / \mathrm{g} /, / \mathrm{m} /, / \mathrm{n} /$.

4. Duração - diferença entre /s/, /S/, / z/, /Z/ e as outras 12 consoantes. Essas quatro consoantes são longas, intensas e de alta frequência.

5. Ponto de articulação - esse traço determina onde ocorre na boca a maior constrição de passagem vocal. Têm-se então três grupos: $/ \mathrm{p} /, / \mathrm{b} /, / \mathrm{f} /$, $/ \mathrm{v} /, / \mathrm{m} /$ como frontais; $/ \mathrm{t} /, / \mathrm{d} /, / \mathrm{tS} /, / \mathrm{s} /$, /dZ/,/z/,/n/ como médias e /k/, /g/,/S/, /Z/ como consoantes posteriores.

Cada uma das listas apresentadas, tanto no teste IPRF quando no teste $F / R$, continha 36 apresentações (fonemas consoantes). Assim, a pontuação de $100 \%$ seria referente ao acerto de todos estes fonemas e conforme diminui o número de acertos, diminui a porcentagem.

\section{RESULTADOS}

Em todo o trabalho, as análises estatísticas foram feitas considerando-se ambas as orelhas, totalizando 98 orelhas no grupo estudo (GE) e 12 no grupo controle (GC), por não haver diferenças significantes entre os resultados das orelhas ( $\mathrm{p}$-valor $>0,05$ ).

Nos gráficos 1, 2, 3 e 4 são mostradas a análise da comparação entre a proporção da presença e da ausência das omissões e de cada um dos tipos de trocas (outras trocas, duração, fricção, ponto de articulação e vozeamento) nos testes IPRF e F/R para o GC e em seguida para o GE. 
Gráfico 1 - Comparação entre a proporção de respostas para cada tipo de troca ou omissão apresentada no teste IPRF para o grupo controle. 2007.

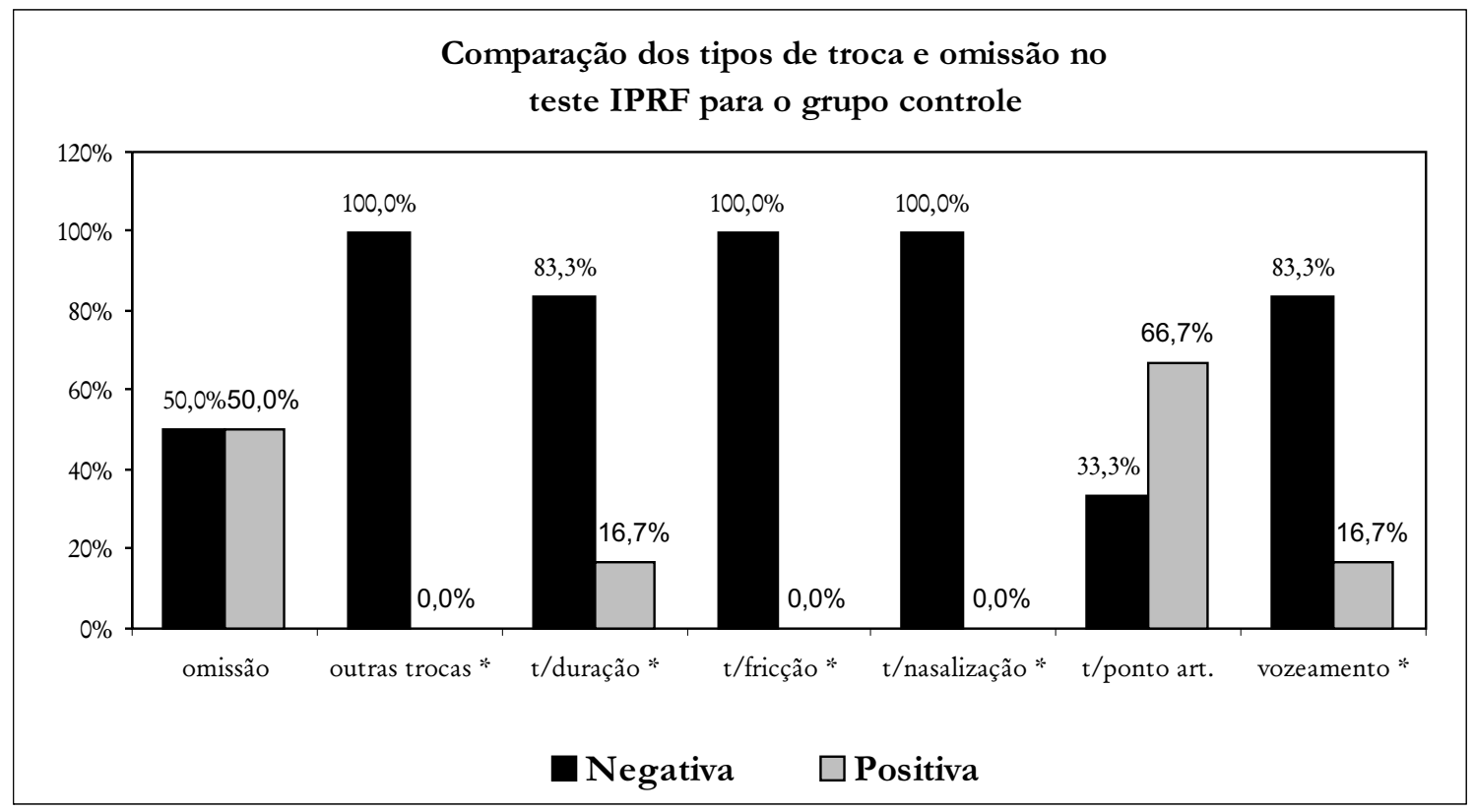

No gráfico acima, a diferença entre a proporção da presença e da ausência de troca foi estatisticamente significante ( $\mathrm{p}$-valor $<0,05)$ para os seguintes tipos de trocas: outras trocas, duração, fricção, nasalização e vozeamento.

Gráfico 2 - Comparação entre a proporção de respostas para cada tipo de troca ou omissão apresentada no teste F/R para o grupo controle. 2007.

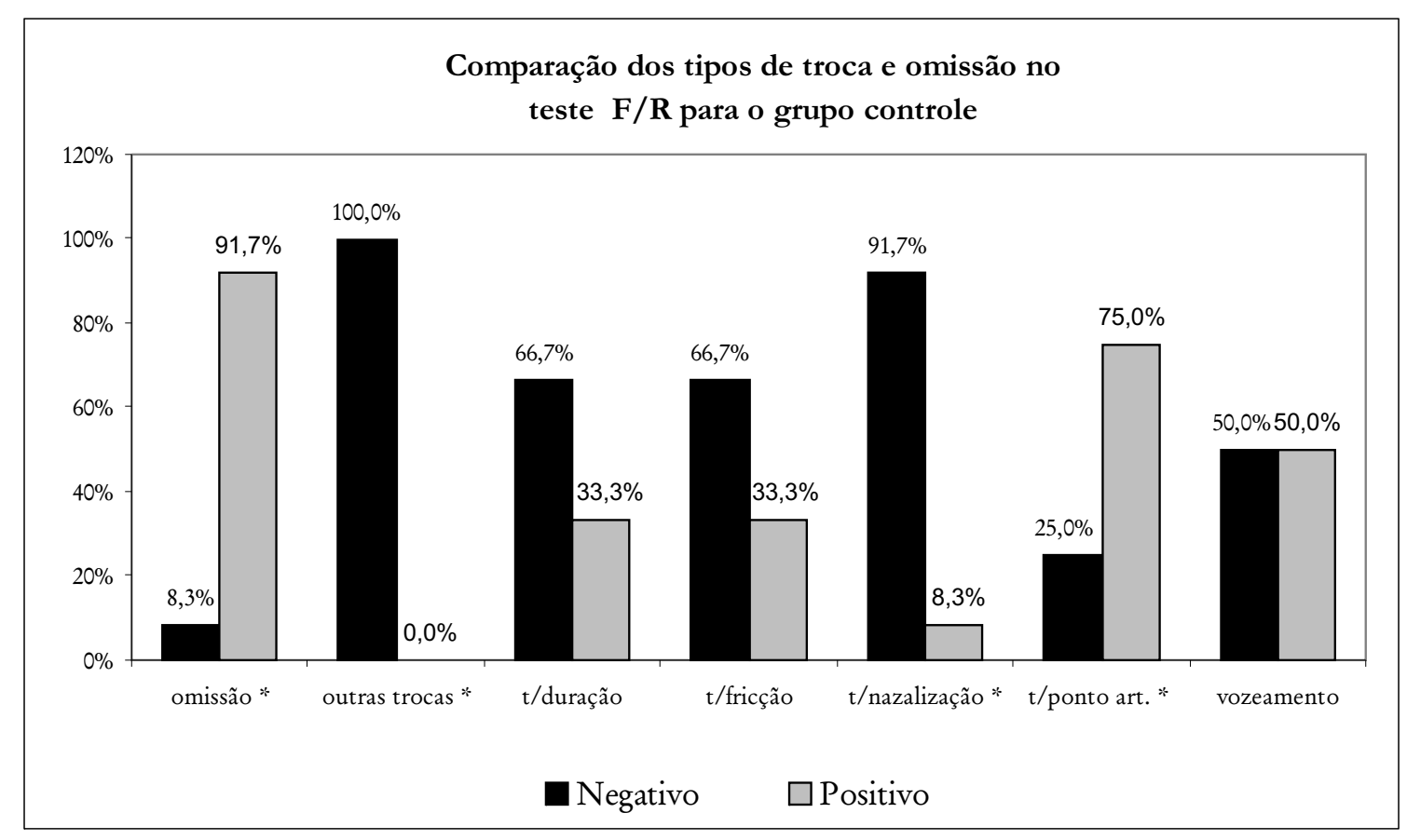


No gráfico 2, nas trocas do tipo outras trocas, nasalização e ponto articulatório e também na omissão, as diferenças entre as proporções de resposta foram estatisticamente significante ( $\mathrm{p}$-valor $<0,05)$.

Gráfico 3 - Comparação entre a proporção de respostas para cada tipo de troca ou omissão apresentada no teste IPRF para o grupo estudo. 2007.

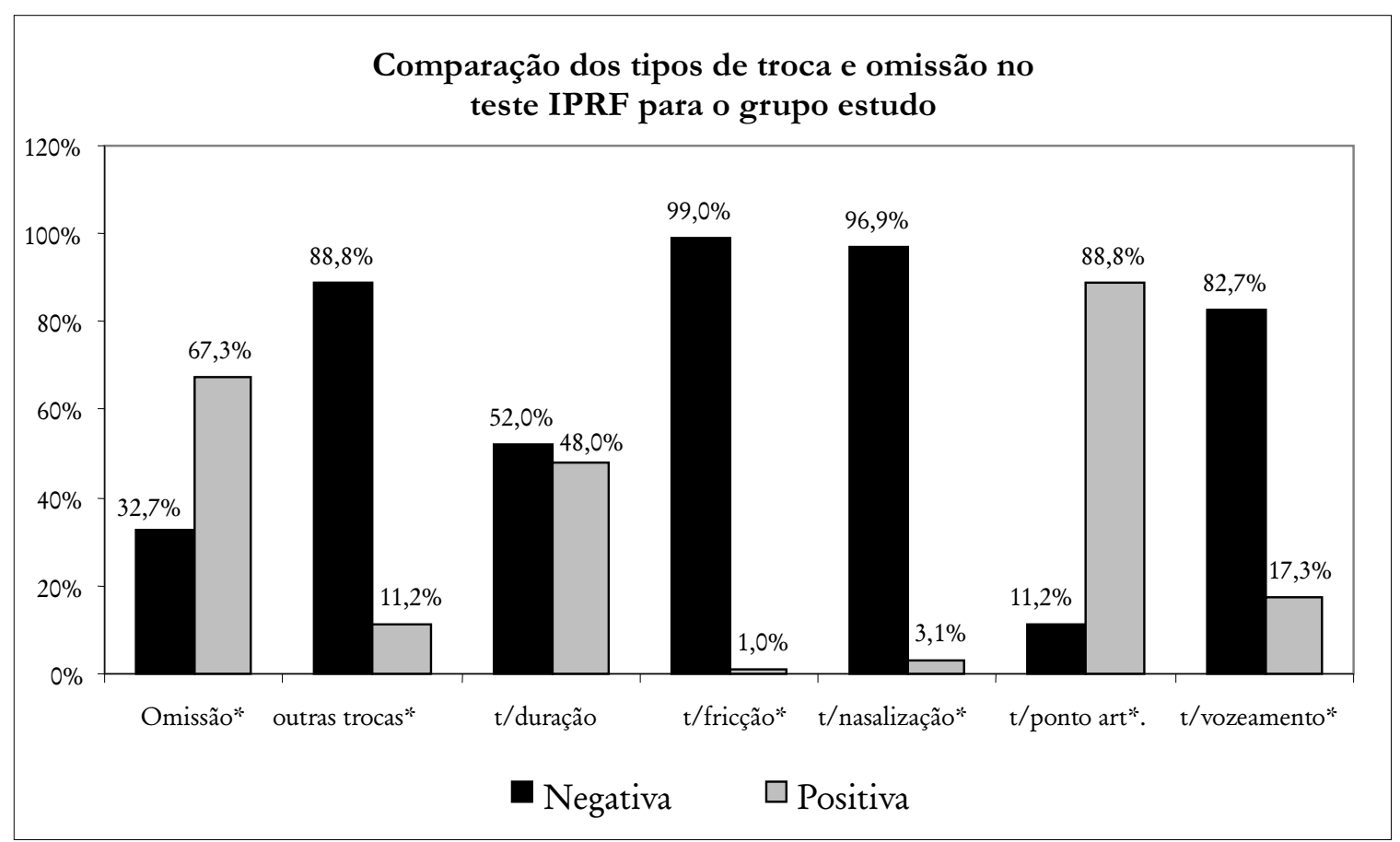

No gráfico 3, somente na troca de duração a negativa não foi estatisticamente significante (pdiferença entre proporção de resposta positiva e valor $>0,05)$. 
Gráfico 4 - Comparação entre a proporção de respostas para cada tipo de troca ou omissão apresentada no teste F/R para o grupo estudo. 2007.

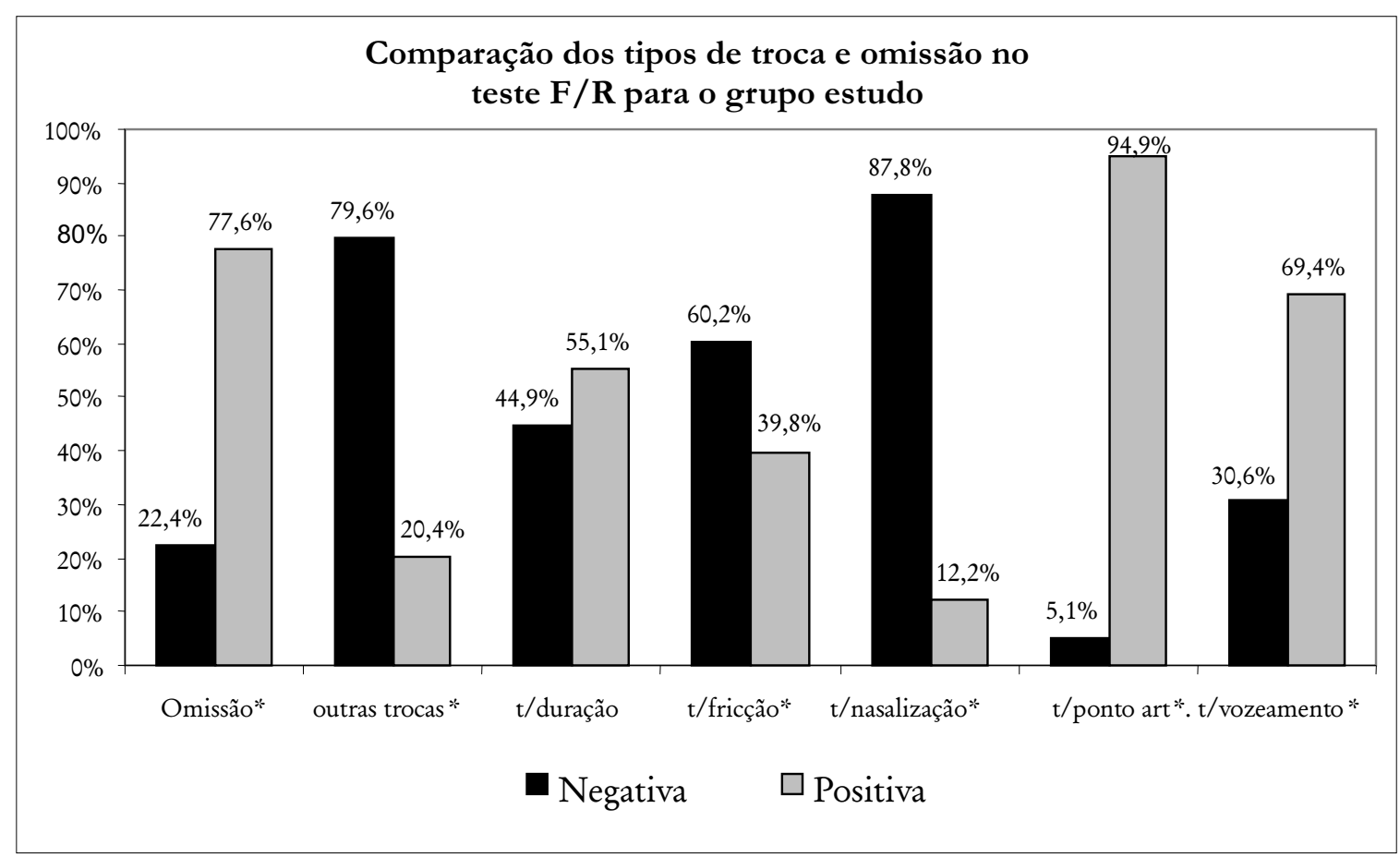

No gráfico 4, somente na troca de duração a diferença entre proporção de resposta positiva e negativa não foi estatisticamente significante ( $\mathrm{p}$ valor $>0,05)$.

Além da análise acima descrita, foi realizada uma análise qualitativa das trocas ocorridas por meio da Matriz de Confusão, e também das omissões observadas. Para cada item considerado, primeiramente serão mostrados os dados referentes ao GC e, a seguir, do GE. Entretanto, antes do início desta análise, alguns comentários se fazem necessários.

As trocas e omissões consideradas com a utilização da Matriz de Confusão são relativas aos fonemas consonantais, não sendo consideradas as trocas que envolvem as vogais ou sons que a representam, ou mesmo o acréscimo de fonemas (exemplos: troca de "tem" por "tim" e "cal" por "carro"). Este critério foi admitido pelos autores que criaram a Matriz de Confusão, já que as consoantes são mais passíveis de confusão e também por serem mais importantes para a inteligibilidade de fala.
Com relação aos tipos de troca (duração, fricção, nasalização, ponto de articulação e vozeamento), em alguns casos ela podia ser considerada em mais de uma categoria, uma vez que os fonemas envolvidos tinham traços de mais de uma delas (ex.: troca de /dz/ por /Z/ que pode ser considerada como troca de duração ou ponto de articulação). Sendo assim, para facilitar a padronização dos dados, nestes casos, apenas uma das categorias foi escolhida e foi mantido o mesmo padrão de escolha em casos que fossem semelhantes.

Considerando as omissões na condição do teste IPRF, no GC não ocorreram omissões de vocábulos, mas dos seguintes fonemas: /p/ e /b/ na posição inicial e /s/ na posição final. Para o GE, foram omitidos os vocábulos "rir" e "sul" e os fonemas /p/ e /b/ na posição inicial e /s/ na posição final.

$\mathrm{Na}$ condição do teste $\mathrm{F} / \mathrm{R}$, no GC não ocorreram omissões de vocábulos, mas dos fonemas /p/ e /b/ na posição inicial e /s/e /R/ 
na posição final. No GE ocorreram omissões dos vocábulos "rir", “faz”, "sul”, "nu”, "ler”, “jaz”, "rol”, "boi”, “dor”, “dil”, “mil”, "lhe”, “cão”, “cal”, "tem" e "vai" e dos fonemas /s/ e /R/ na posição final e os plosivos /p/ e /b/ na posição inicial.

Quanto às trocas não possíveis de classificação e que foram categorizadas como outras trocas, vale ressaltar que somente ocorreram no grupo estudo, sendo exemplos delas: troca de "tom" por "cão" (no que se refere ao fonema /m/), "jaz" por "jão" (fonema /z/) e "gim" por "gil” (fonema /m/).
A seguir, são exemplificadas as trocas ocorridas, sendo a ordem de apresentação dos vocábulos dos que foram trocados mais frequentemente para os que ocorreram menos vezes ou ainda uma única vez. Também para cada um dos vocábulos, a ordem das trocas exemplificadas seguiu o mesmo padrão.

No quadro 1, são mostradas as trocas para o teste IPRF e F/R para o Grupo Controle (GC). A média de ocorrência de trocas foi de 3,25\% para o teste IPRF e $8,54 \%$ para o $F / R$.

Quadro 1 - Caracterização dos tipos de trocas apresentadas nos testes IPRF e F/R para o GC. 2007.

\begin{tabular}{|l|l|l|}
\hline Vocábulo apresentado & Trocas IPRF & Trocas F/R \\
\hline Rir & fir, vir, fiz & fir, fre \\
\hline Dil & til & til \\
\hline Lhe & le, ge & ve, le, ler, que \\
\hline Faz & face & paz \\
\hline Rol & ----- & sol, foi \\
\hline Pus & ----- & urso \\
\hline Boi & ----- & oito \\
\hline Jaz & ------ & jarci \\
\hline Nu & ---- & til \\
\hline Gim & ---- & til \\
\hline
\end{tabular}

A seguir, no quadro 2, são mostradas as trocas para o teste IPRF e F/R para o Grupo Estudo
(GE). A média da ocorrência de trocas foi de $6,5 \%$ para o teste IPRF e 13,48\% para o F/R. 
Quadro 2 - Caracterização dos tipos de trocas apresentadas nos testes IPRF e F/R para o GE. 2007.

\begin{tabular}{|c|c|c|}
\hline Vocábulo apresentado & Trocas IPRF & Trocas F/R \\
\hline rir & $\begin{array}{l}\text { fir, xis, sir, fiz, ser, vir, firs, fil, fez, } \\
\text { fieci, Fiat, risi, fre, xir, xi }\end{array}$ & $\begin{array}{l}\text { fir, vir, ser, sir, xis, feliz, ris, ver, } \\
\text { frirs, firso, firsi, fil, se, féis, Fiat, } \\
\text { fix, fri, frit, Felipe, fer }\end{array}$ \\
\hline dil & til, gil & til, gil, xil \\
\hline lhe & ge, ve, ler, le, vir, guet & ge, ler, ve, ver, le, rir, vem, lha, til \\
\hline faz & face, fax, jacs, fati & $\begin{array}{l}\text { paz, pa, par, fax, ar, pai, face, } \\
\text { faca, puz, va, mais }\end{array}$ \\
\hline rol & sol & sol, som, sono, tiol, cão, zum \\
\hline pus & $\begin{array}{l}\text { urs, urso, rus, luz, ushi, ursi, cus, } \\
\text { fus, usi, plus }\end{array}$ & $\begin{array}{l}\text { urso, luz, urs, plus, sus, cus, curs, } \\
\text { russo }\end{array}$ \\
\hline tom & cão, com, pão, por & cão, som \\
\hline zum & jum & gim, sum, gil, tim, gi, xi \\
\hline nha & ner, mir, mer, na & $\begin{array}{l}\text { mer, mel, ler, mulher, miau, } \\
\text { minha, val, me, mé, leste, val, } \\
\text { nhé, mil, né }\end{array}$ \\
\hline gim & ge, xi, xim & xi, xis, xim, ge, tim, xe, xir \\
\hline mil & fil, vil & \\
\hline $\mathrm{nu}$ & lu, meu & xil, um, deu, lu \\
\hline til & xil, xir & di, doze, gil, xir \\
\hline dor & dois, doce & doce, sem, dos, flor, dois \\
\hline seis & se & se, sir \\
\hline ler & lessi & faz, ver \\
\hline boi & oito & oito, foi, mil \\
\hline jaz & chá & ter, chá, seje, lha \\
\hline cal & tal & ---- \\
\hline vai & ----- & pai, cai, xai \\
\hline mil & ler, livro & ler, livro \\
\hline tem & -.-- & ter, zum, véu \\
\hline sul & - & pus, ler \\
\hline
\end{tabular}




\section{DISCUSSÃO}

De maneira geral, o que pode ser observado nos resultados foi um pior desempenho geral para o grupo estudo, principalmente quando apresentado o estímulo auditivo junto com o ruído competitivo. Estes dois fatos são justificados, uma vez que os idosos do grupo estudo eram todos portadores de perda auditiva, ainda que nas frequências altas, e com idade mínima, média e máxima superiores a do grupo controle.

Os estudos da literatura que compararam o desempenho entre grupos também relataram diferenças entre eles, com pior desempenho para os idosos sadios (quando comparados com jovens) ou para os idosos portadores de perda auditiva e um impacto negativo da presença do ruído junto com o material de fala. Entretanto, utilizaram grupos de sujeitos com características audiométricas e de idade distintas e/ou metodologias que diferiram do presente trabalho, inviabilizando maiores comparações ${ }^{8,12,14,15,23}$.

Quanto à comparação entre a proporção de respostas (positiva ou negativa) para cada tipo de troca e para a omissão, e considerando o GC na condição do teste IPRF (Gráfico 1), somente não ocorreu diferença estatisticamente significante para a troca do tipo ponto articulatório e para a omissão, sendo este tipo de troca a que mais ocorreu seguida da seguida da omissão. A troca de ponto articulatório parece revelar que, neste caso, os traços acústicos que diferenciam um fonema do outro seriam talvez mais sutis e, portanto, mais dificilmente percebidos pelos idosos, mesmo para aqueles com boa audição periférica e sem a presença do ruído. No caso da omissão, duas hipóteses podem ser levantadas: primeiro, um comportamento do sujeito de se omitir, ou seja, não arriscar diante da incerteza do correto reconhecimento e outra conjectura seria a necessidade de mais tempo para processar quais seriam as possibilidades diante da dúvida. Com a continuidade do teste, o tempo disposto não seria suficiente para o sujeito emite algum vocábulo e, então, ele passa a ter atenção ao próximo vocábulo. Para as demais trocas, a diferença foi estatisticamente significante, com maior proporção para a ausência das trocas. Um aspecto interessante a ser observado é que, para as trocas do tipo outras trocas, fricção e nasalização não houve erros, demonstrando que trocas incomuns, bem como as características acústicas dos sons de fonemas que envolvem a fricção e a nasalização foram suficientes para os idosos, sem perda auditiva, perceberem e identificarem corretamente os sons na situação de escuta sem ruído.

No gráfico 2, nas trocas do tipo outras trocas, nasalização e ponto articulatório e também na omissão, as diferenças entre as proporções de resposta foram estatisticamente significantes ( $\mathrm{p}$ valor $<0,05)$.

Ainda considerando o GC, quanto ao desempenho no teste F/R (Gráfico 2), ocorreu diferença estatisticamente significante para as trocas do tipo outras trocas, nasalização e ponto articulatório, e também para a omissão. Quanto à proporção de respostas, foi maior a ocorrência da omissão seguida da troca de ponto articulatório e menor ocorrência das trocas do tipo outras trocas e de nasalização. Vale ressaltar que, na situação da presença do ruído competitivo, somente na troca do tipo outras trocas houve $100 \%$ de acerto, demonstrando que a presença do ruído dificultou a identificação dos sons e que somente trocas mais extravagantes ou menos comuns (outras trocas) deixaram de ocorrer, o que pode ser visualizado pelo aumento de amplitude das colunas cinzas e diminuição das pretas no Gráfico 2, quando comparado com o Gráfico 1.

O fato de o GC ter apresentado um pior desempenho na situação de teste com a presença de um ruído competitivo revela dois aspectos importantes. Primeiro, o efeito negativo do ruído no reconhecimento de fala, mesmo para idosos sem perda auditiva, fato já observado na literatura ${ }^{8,11,12}$. O segundo aspecto está relacionado ao fato de o envelhecimento não se limitar apenas ao órgão de Corti (audição periférica), mas a todo o sistema auditivo ${ }^{24-26}$. Assim, mesmo a ausência de uma perda auditiva, detectada no exame de audiometria tonal, não garante um desempenho adequado quanto ao reconhecimento de fala. 
Quando analisamos o GE na situação do teste IPRF (Gráfico 3), somente na troca do tipo duração a diferença entre a proporção da ausência e da presença da troca não foi estatisticamente significante. A maior proporção da ocorrência das trocas aconteceu na de ponto articulatório e na omissão de resposta. Este padrão de resposta foi igualmente presente no grupo controle, revelando mais uma vez que o traço linguístico que determina onde ocorre na boca a maior constrição de passagem vocal (ponto articulatório) foi o mais dificilmente percebido pelo idoso e, portanto, mais passível de confusão para uma correta identificação. Talvez uma análise espectral dos sons envolvidos neste tipo de troca pudesse esclarecer o porquê deste ocorrido. Ressalta-se que em nenhum dos casos houve $100 \%$ de acerto, mostrando que a presença da perda auditiva foi suficiente para impedir que o idoso identificasse corretamente os sons, mesmo em situação ideal de escuta (sem ruído competitivo).

$\mathrm{Na}$ condição do teste $\mathrm{F} / \mathrm{R}$ para o GE (Gráfico 4), assim como na condição do teste IPRF, somente na troca do tipo duração a diferença entre a proporção da ausência e da presença da troca não foi estatisticamente significante. $\mathrm{Na}$ troca de ponto articulatório, vozeamento e na omissão, houve maior proporção de ocorrência das trocas e, para os demais tipos de troca, foi maior a proporção de ausência das trocas. Assim como na condição do teste IPRF, no teste $\mathrm{F} / \mathrm{R}$ não houve $100 \%$ de acerto em nenhuma das opções de troca e, além disso, o índice positivo para as trocas foi ainda maior (colunas cinzas). Desta forma, a existência da perda auditiva associada à presença do ruído competitivo é mais do que suficiente para impedir a correta identificação dos sons.

$\mathrm{Na}$ literatura, os estudos localizados não contemplaram tipo de análise semelhante à do presente trabalho para que se pudessem estabelecer comparações. Entretanto, três estudos fizeram algum tipo de análise quanto às trocas ocorridas com o material de fala.

Schochat ${ }^{11}$ revelou em seu estudo que as alterações que ocorreram com maior significância foram as trocas de /t/ por /s/ e /f/ por /p/, que seriam classificadas como trocas de fricção, e /d/ por / $\mathrm{t} /$, que seriam classificadas como trocas de vozeamento se a análise fosse feita pela MC.

No caso do estudo apresentado neste artigo, as trocas do tipo fricção e vozeamento não foram as que mais frequentemente ocorreram em nenhum dos grupos e em nenhuma das condições, mas nas possibilidades de troca apresentaram uma ocorrência considerável. A troca do tipo fricção apresentou baixíssima ocorrência na condição do teste IPRF para os dois grupos, da mesma forma que outros tipos de troca e, na presença do ruído, ocupou a terceira posição para os idosos sem perda auditiva periférica e a quarta posição para os idosos com perda. A troca do tipo vozeamento foi a segunda que mais ocorreu na condição do teste IPRF para os dois grupos e também na condição com ruído para o grupo controle, passando a ocupar a terceira posição, nesta mesma condição, para o grupo estudo.

As diferenças entre o trabalho de Schochat ${ }^{11} \mathrm{e}$ o presente estudo podem ter ocorrido por diferenças na metodologia segundo a qual o teste foi aplicado (diferentes tipos de ruído e diferentes relações sinal/ruído), bem como diferenças entre os grupos de idosos avaliados no que se refere aos limiares tonais e características pessoais dos grupos avaliadas (idosos mais ou menos ativos e com rotinas distintas).

Martini et al. ${ }^{17}$ relataram a ocorrência de um elevado índice de trocas referidas como completa distorção e menor índice de trocas envolvendo fonemas de diferentes categorias e menor ainda envolvendo fonemas da mesma categoria. Entretanto, não foram exemplificadas as trocas ocorridas dentro destas três categorias, impossibilitando maiores comparações com os dados apresentados no presente estudo.

Shrivastav et al..$^{18}$ observaram desempenhos melhores em tarefas de identificação de fricativas do que de plosivas. No estudo apresentado, não foi feita uma análise semelhante para uma comparação mais direta. Todavia, ao se observar 
os resultados do estudo (gráficos de 1 a 4), é possível identificar, de maneira geral, um índice elevado de acerto quando considerada a troca do tipo fricção.

Com relação à análise qualitativa das omissões e trocas apresentadas pelos grupos controle e estudo, dados interessantes serão discutidos a seguir, mas inicialmente alguns pontos merecem ser comentados.

A utilização da análise por meio da Matriz de Confusão considera as trocas apresentadas relacionadas aos fonemas consonantais somente e desconsidera o acréscimo de outros sons. Isto demonstra a limitação deste tipo de análise, pois apesar de os fonemas consonantais serem mais importantes para a compreensão dos sons de fala, uma troca envolvendo uma vogal, ou mesmo o acréscimo de algum som, resulta em erro, dificultando a efetividade e eficácia de um diálogo.

Com relação às omissões de vocábulos, é interessante observar que elas não ocorreram no GC, revelando que a normalidade dos limiares tonais favoreceu a escuta e compreensão dos vocábulos apresentados, ou pelo menos, parte deles. Já para o GE, ocorreram omissões de vocábulos, mas sobretudo na condição do teste $F / R$, revelando que a presença de uma perda auditiva, ainda que pequena e concentrada nas frequências agudas, acarreta diminuição ou perda das pistas acústicas de fonemas para sua correta identificação.

Quanto à omissão de fonemas, o padrão de normalidade dos limiares tonais apresentado pelo GC não impediu que alguns fonemas fossem omitidos, mesmo na ausência de um estímulo competitivo. Esta situação pode ter ocorrido devido ao tipo de material utilizado, isto é, monossílabos (unidade de menor valor semântico) e também à deterioração do processamento central, fato já previamente observado na literatura ${ }^{26-30}$.

Ainda considerando a omissão de fonemas, mas com relação ao GE, o que o diferenciou do GC foi o número superior da frequência das omissões e o maior impacto na condição do teste F/R. Um aspecto interessante observado foi a semelhança destas omissões com os plosivos /p/ e /b/ na posição inicial e o arquifonema /s/ na final, principalmente na condição do teste $\mathrm{F} / \mathrm{R}$.

$\mathrm{Na}$ literatura, somente foi localizado o estudo de Schochat ${ }^{11}$, que realizou semelhante forma de análise dos erros com o relato da ocorrência da omissão do arquifonema /s/ em posição final, tanto no grupo de idosos sem perda auditiva como nos portadores de perda auditiva.

Com relação à omissão do arquifonema /s/ para o grupo estudo, uma possível explicação residiria na frequência deste fonema (próximo de $6 \mathrm{kHz})^{31}$, sendo que todos os idosos deste grupo apresentavam perda nas frequências agudas, no mínimo nas frequências de 6 e $8 \mathrm{kHz}$.

Com relação às trocas apresentadas para o GC e GE na condição do teste IPRF, é possível observar que os vocábulos trocados ("rir", "dil”, "lhe" e "faz"), bem como as trocas em que resultaram (exemplos: "rir" por "fir" e "lhe" por "ge"), realizadas no primeiro grupo, se repetem no segundo, além do acréscimo de outros vocábulos (exempos "pus" e "rol"). Vale ressaltar que no $\mathrm{GE}$ a variedade e a quantidade das trocas foi maior que no GC.

$\mathrm{Na}$ condição de teste do $\mathrm{F} / \mathrm{R}$, houve aumento do número de vocábulos que foram erroneamente repetidos tanto para o GC quanto para o GE, o que pode ser observado nos quadros 1 e 2 . Com relação à semelhança das trocas entre os grupos, outro autor ${ }^{11}$ também observou este tipo de situação, na qual as trocas foram semelhantes para os grupos avaliados (jovens e idosos sem e com perda auditiva), levantando a hipótese de que são utilizadas as mesmas pistas acústicas para a decodificação do sinal acústico.

No que diz respeito ao motivo de alguns vocábulos terem sido mais trocados do que outros, pode-se inferir o grau variado de dificuldade que os mesmos oferecem. Como previamente observados por Schochat ${ }^{11}$, que utilizou similar lista de vocábulos, alguns são amplamente utilizados na língua portuguesa, e 
outros não têm sentido para a maioria da população.

Com relação à ocorrência de algumas trocas passíveis de explicação, tem-se as pistas acústicas que caracterizam os fonemas como na diferença de duração /f/ e do /b/ e também pelo aspecto semântico, como na troca de "rol" por "sol", entre outras. Semelhante consideração foi realizada em um estudo prévio ${ }^{11}$.

A respeito das trocas realizadas nas quais o sujeito substitui por um vocábulo mais familiar, um estudo ${ }^{16}$ que também utilizou a Matriz de Confusão observou que a utilização de monossílabos como estímulo de fala fez com que o paciente respondesse com outra palavra que nem sempre refletia a troca, devido a características acústicas dos fonemas, estando o erro sujeito a interferências do contexto semântico. Assim, diante da dificuldade de compreender o que escutou, a pessoa busca palavras com significado para ela.

Entre as trocas realizadas, algumas merecem ser destacadas, por não serem passíveis de explicação. Elas podem ter ocorrido pelo indivíduo ter tentado adivinhar o que ouviu e ocorreram mais frequentemente no GE e na condição do teste F/R, como nos exemplos, "fiat" e "Felipe" para "rir", e "livro" para "mil". Vale ressaltar que estas trocas foram pouco frequentes de maneira geral, com exceção de algumas delas, como "pus" para "urso" e "oito" para "boi".

Schochat ${ }^{11}$ também observou semelhante aspecto sem também conseguir decifrar o motivo para tal ocorrido. Talvez uma análise do espectro de frequência destes vocábulos possa elucidar esta situação que se repetiu em diferentes estudos.

O presente estudo revelou dados importantes quanto às habilidades auditivas de ouvintes idosos no que se refere à percepção auditiva dos sons de fala, bem como à interferência da presença do ruído concomitante. Além disso, demonstrou a necessidade de outros estudos que realizem uma análise mais minuciosa, considerando os erros apresentados nos testes de fala.

\section{CONCLUSÃO}

A análise qualitativa e quantitativa dos erros apresentados em um teste de fala na presença de ruído competitivo, em idosos, permitiu-nos chegar às seguintes conclusões:

- A presençade limiares tonais dentrodos padrões de normalidade não impede que idosos apresentemerros no reconhecimento de fala.

- A presença do ruído interfere negativamente no reconhecimento dos sons de fala, principalmente quando o idoso apresenta perda auditiva.

- Os fonemas plosivos /p/ e /b/, na posição inicial de vocábulos, e o arquifonema /s/, na posição final, foram os mais frequentemente omitidos, principalmente na presença do ruído.

- Os vocábulos "rir”, “dil”, "lhe”, “faz” e "rol" foram os mais frequentemente trocados, principalmente na presença do ruído. 


\section{REFERENNCIAS}

1. Instituto Brasileiro de Geografia e Estatística. [ acesso em 12 jan 2009]. Disponível em http:// www.ibge.gov.br.

2. Viude A. Fatores associados a presbiacusia em idosos [Tese]. São Paulo: Universidade de São Paulo; 2002.

3. Weinstein BE. Presbiacusia. In: Katz J. Tratados de audiologia clínica. $4^{\text {a }}$ Edição. São Paulo: Manole, 1999, p.562-76.

4. Gates GA, Mills JH. Presbycusis. Lancet 2005; 366 (9491):1111-20.

5. Rados C. Sound advice about age-related hearing loss. FDA Consum 2005. May-Jun; 39(3):20-7.

6. Calais LL, Russo ICP, Borges ACLC. Desempenho de idosos em um teste de fala na presença de ruído. Pro-Fono [online] 2008; 20(3):147-52.

7. Pereira LD, Shochat E. Fala com ruído (teste 1). In: Pereira LD, Shochat E. Processamento Auditivo Central- manual de avaliação. São Paulo: Lovise; 1997. p. 99-102.

8. Duarte VG. O efeito do ruído na percepção de fala de indivíduos ouvintes jovens e idosos. [Dissertação]. São Paulo: Pontifícia Universidade Católica de São Paulo; 1998.

9. Kenyon EL, Leidenheim SE, Zwillenberg S. Speech discrimination in sensorineural hearing loss patient: how is it affected by background noise? Mil Med 1998;163(9):647-50.

10. Pichora-Fuller MK, Souza PE. Effects of aging on auditory processing of speech. Int J Audiol. 2003;42(Suppl.2S):11-26.

11. Schochat E. Percepção de fala: presbiacusia e perda auditiva induzida por ruído. [Tese de Doutorado]. São Paulo: Faculdade de Filosofia, Letras e Ciências Humanas-Universidade de São Paulo; 1994.

12. Pichora-Fuller MK, Schneider BA, Daneman M. How young and old adults listen and remember speech in noise. J. Acoust. Soc. Am. 1995; 97(1): 593-608.

13. Studebaker GA, Sherbecoe RL, McDaniel DM, Gwaltney CA. Monosyllabic word recognition at higher-than-normal speech and noise levels. J. Acoust. Soc. Am. 1999; 105(4): 2431-44.

14. Caporali SA, Silva JA. Reconhecimento de fala no ruído em jovens e idosos com perda auditiva. Rev Bras Otorrinolaringol. 2004; 70(4): 525-32.
15. Souza PE, Boike KT, Witherell K, Tremblay K. Prediction of speech recognition from audibility in older listeners with hearing loss: efects of age, amplification, and background noise. J. Am Acad Audiol. 2007; 18 (1): 54-65.

16. Helou LF, Novaes BC. Utilização da matriz de confusão na indicação de aparelho de amplificação sonora individual. Distúrb. Comum. 2005; 17(2): 203-13.

17. Martini A, et al. Heating in the elderly: a population study. Audiology. 2001; 40(6): 285293.

18. Shrivastav MN, Humes LE, Kewlev-Port D. Individual differences in auditory discrimination of spectral shape and speech-identification performance among elderly listeners. J. Acoust. Soc. Am. 2006; 119(2):1131-42.

19. Brasil. Lei n. 10.741 de $1^{\circ}$ de outubro de 2003. Dispõe sobre o Estatuto do Idoso e dá outras providências. Diário Oficial da República Federativa do Brasil, Brasília (DF); 2003 Out 3.

20. Jerger J. Clinical experience with impedance audiometry. Arch Otolaryngol. 1970; 92: 311-24.

21. International Standards Organization. Acoustics - Audiometric test methods. Part I: Basic pure tone air and bone conduction threshold audiometry. Geneva, Switzerland. ISO 8253-1; 1989.

22. Silva AM, Gordo A, Pereira LD. Índice perceptual de reconhecimento de fala com e sem ruído em indivíduos com perda condutiva e neurossensorial - estudo comparativo. Acta AWHO. 1997; 16(4):174-8.

23. Bertachini L, Gonçalves MJ. Comunicação na terceira idade. Mundo saúde 2002; 26(2):483-89.

24. Céspedes JHH. Causas da Hipoacusia Sensorioneural. Rev. Méd. Clín. Condes. 2003: 14(1); [cerca de 10p.]. Disponível em: http://www.clinicalascondes.cl/ area_academica/ Revista_Medica_Enero_2003/ articulo_002.htm

25. Chisolm TH, Willot JF, Lister JJ. The aging auditory system: anatomic and physiologic changes and implications for rehabilitation. Int $\mathrm{J}$ Audiol. 2003; 42: 2(S):3-10.

26. Pinheiro MMC, Pereira LD. Processamento auditivo em idosos: estudo da interação por meio de testes com estímulos verbais e não verbais. Rev Bras Otorrinolaringol. 2004; 70(2):209-14. 
27. Golding M, Carter N, Mitchell P, Hood LJ. Prevalence of central auditory processing (CAP) abnormality in an older Australian population: the blue mountain hearing study. J Am Acad Audiol. 2004; 15(9): 633-42.

28. Gates GA, Mills JH. Presbycusis. Lancet. 2005; 366 (9491): 1111-20.
29. Mazelová J, Popelar J, Syka J. Auditory function in presbyacusis: peripheral vs. central changes. Exp Gerontol. 2003; 38(12):87-94.

30. Russo ICP, Behlau M. Percepção da fala: análise acústica do Português Brasileiro. São Paulo: Lovise; 1993. p. 57.

Recebido: 26/5/2009

Revisado: 04/2/2010

Aprovado: 30/8/2010 


\section{Anexo 1}

Universidade Federal de São Paulo - Escola Paulista de Medicina

Departamento de Fonoaudiologia

Disciplina dos Distúrbios da Comunicação Humana

Nome:

Idade:

Data:

$\operatorname{IPRF}($ )

$\mathrm{F} / \mathrm{R}($ )

\begin{tabular}{|c|c|c|c|c|c|}
\hline $\mathbf{N}$ & D 1 & D 2 & D 3 & D 4 & $\%$ \\
\hline 1 & TIL & CHÁ_ & DOR & JAZ_ & 96 \\
\hline 2 & GÁS_ & DOR_ & $\mathrm{BOI}$ & CÃO & 92 \\
\hline 3 & $\mathrm{ROL}_{-}$ & MIL & TIL & $\mathrm{CAL}_{-}$ & 88 \\
\hline 4 & PUS & $\mathrm{TOM}_{2}$ & $\mathrm{ROL}_{-}$ & $\mathrm{BOI}$ & 84 \\
\hline 5 & FAZ & ZUM & GIM & $\mathrm{NU}_{-}$ & 80 \\
\hline 6 & GIM & MEL_ & $\mathrm{CAL}_{2}$ & FAZ & 76 \\
\hline 7 & RIR_ & TIL_ & NHÁ & GIM & 72 \\
\hline 8 & $\mathrm{BOI}$ & GIM & CHÁ & PUS & 68 \\
\hline 9 & VAI_ & DIL_ & $\mathrm{TOM}_{-}$ & SEIS_ & 64 \\
\hline 10 & MEL & $\mathrm{NU}_{-}$ & SUL_ & NHÁ & 60 \\
\hline 11 & $\mathrm{NU}_{-}$ & PUS & TEM_ & MIL_ & 56 \\
\hline 12 & LHE_ & NHÁ & PUS & TEM & 52 \\
\hline 13 & $\mathrm{CAL}_{-}$ & SUL & $\mathrm{NU}_{-}$ & ZUM & 48 \\
\hline 14 & MIL & JAZ & CÃO & TIL & 44 \\
\hline 15 & TEM & $\mathrm{ROL}_{-}$ & VAI & LHE_ & 40 \\
\hline 16 & DIL_ & TEM & MEL & SUL & 36 \\
\hline 17 & DOR & FAZ & RIR_ & CHÁ & 32 \\
\hline 18 & CHÁ & LHE & GÁS & $\mathrm{ROL}_{-}$ & 28 \\
\hline 19 & ZUM & $\mathrm{BOI}$ & ZUM & MEL & 24 \\
\hline 20 & NHÁ & $\mathrm{CAL}_{-}$ & LHE & DOR & 20 \\
\hline 21 & CÃO & RIR_ & MIL & VAI & 16 \\
\hline 22 & $\mathrm{TOM}$ & CÃO & LER_ & DIL & 12 \\
\hline 23 & SEIS & LER_ & FAZ & TOM & 8 \\
\hline 24 & LER_ & VAI & SEIS & RIR_ & 4 \\
\hline 25 & SUL_ & SEIS & DIL & LER & 0 \\
\hline
\end{tabular}

